Gerión. Revista de Historia Antigua

ISSN: 0213-0181

http://dx.doi.org/10.5209/GERI.56959

\title{
Pu(blia) Fulvia Plautilla Augusta. Consideraciones acerca del praenomen de la emperatriz
}

\author{
Rafael González Fernández
}

Recibido: 15 de diciembre de 2015 / Aceptado: 22 de junio de 2016

Resumen. En este trabajo pretendemos demostrar que no hay razones suficientes para considerar que Fulvia Plautilla, hija del prefecto del pretorio Caius Fulvius Plautianus, pudiera portar el praenomen Publia, ya que sólo está atestiguado por algunas monedas de la ceca de Tomis, en Moesia Inferior. Hay que considerar los errores cometidos con bastante frecuencia en las acuñaciones de las cecas orientales. Concretamente en algunas monedas de las ciudades de Thyateira y Acrasus, ambas en Lidia, recibe el cognomen Plautiana. Con respecto al praenomen Iusta que aparece en algunas publicaciones, al menos desde 1767, no hay testimonios coetáneos que atestigüen dicho uso.

Palabras clave: Publia; Iusta; Fulvia; Plautilla; Praenomen; Plauciano.

\section{[en] Pu(blia) Fulvia Plautilla Augusta. Considerations on the Praenomen of the Empress}

\begin{abstract}
In this article we try to demonstrate that there are no sufficient reasons to consider that Fulvia Plautilla, daughter of the Prefect Commander of the Praetorian Guard, Caius Fulvius Plautianus, could used the praenomen Publia, since this praenomen is only attested in some coins which were minted in Tomis, in Moesia Inferior. Furthermore, we should take into account the frequent mistakes made in the Oriental minting. Specifically in some coins from Thyateira and Acraus, both in Lidia, she received the cognomen Plautiana. Regarding the praenomen Iusta that appears in some publications, at least from 1767, there are not contemporary evidence which attest its use.
\end{abstract}

Keywords: Publia; Iusta; Fulvia; Plautilla; Praenomen; Plautianus.

Cómo citar: González Fernández, R. (2017) Pu(blia) Fulvia Plautilla Augusta. Consideraciones acerca del praenomen de la emperatriz, en Gerión 35/1, 157-163.

\footnotetext{
Universidad de Murcia

E-mail: rafaelg@um.es
} 
Fulvia Plautilla ${ }^{2}$ y su padre C. Fulvius Plautianus, ${ }^{3}$ prefecto del pretorio del emperador Septimio Severo, procedían de Leptis Magna aunque la familia pudo tener origen itálico. ${ }^{4}$ Contrajo matrimonio con Marcus Aurelius Antoninus, Caracalla, el hijo mayor de Severo en abril de 202,5 si bien recibió el título de Augusta algunos meses antes de la boda, concretamente tras la celebración de los sponsalia. ${ }^{6}$ En 205, tras la eliminación de su padre, fue enviada al exilio a las islas Lípari, en Sicilia, junto con su hermano Caius Fulvius Plautius Hortensianus. ${ }^{7}$ Finalmente en 211, poco después de la muerte de Septimio Severo, ambos hermanos fueron asesinados por orden de Caracalla. La damnatio memoriae que sufrió la emperatriz, posiblemente más intensa tras su muerte que cuando fue exiliada en 205 , no fue tan minuciosa como la del padre, ya que de ella se conservan algunos retratos. ${ }^{8}$

Normalmente la emperatriz es mencionada en las publicaciones simplemente como Fulvia Plautilla, aunque también en ocasiones se hace referencia a un supuesto praenomen: Publia Fulvia Plautilla. Y es precisamente sobre este primer componente del nombre de la emperatriz sobre el que queremos hacer una serie de consideraciones. Las mujeres recibían oficialmente un praenomen el octavo día después de su nacimiento; sin embargo, no se solía ver reflejado en la esfera pública $\mathrm{y}$, aunque no se prodigaban demasiado en los documentos epigráficos, ${ }^{9}$ podemos afirmar que los praenomina de mujeres son conocidos casi exclusivamente a partir de las inscripciones. ${ }^{10} \mathrm{Su}$ desaparición, según Iiro Kajanto ${ }^{11}$ sería consecuencia de la importancia cada vez mayor de la gens y seguramente dejó de tener sentido dado el escaso papel que las mujeres tuvieron en la vida pública, quedando además totalmente en desuso cuando hacia el siglo II a.C. los cognomina se generalizaron. ${ }^{12}$ En general podemos afirmar que tendrían más bien un carácter privado, sobre todo para distinguir a las hermanas y que poco a poco fueron cediendo terreno a los cognomina. Sin embargo, se constata el mantenimiento del praenomen de las mujeres, al menos en las clases altas, en particular en las provincias orientales, en las que seguramente se quiso hacer valer la romanidad adquirida. ${ }^{13}$ Esta práctica

\footnotetext{
KIENAST 2004, 165.

PIR F 554; RE VII 1, 1910, cols. 270-278, $\mathrm{n}^{\circ} 101$ (A. Stein).
}

4 Sobre la familia y origen de los Fulvii africanos puede verse un resumen en GonZÁLEZ FERNÁNDEZ - CONESA NAVARro 2014, 29-30.

5 Birley 2012, 214; Daguet-Gagey 2000, 333; GonzÁlez Fernández - Conesa Navarro 2014, 36-38.

6 Christol - Drew-Bear 1995, 76-78; Christol 1997, 127-136; Conesa Navarro - González Fernández 2016, 136 y 146; GonZÁlez FernáNDEZ - Conesa NAVArro (e.p.).

$P I R^{2}$ F 555; RE VII 1, 1910, col. 278, nr. 102 (A. Stein).

8 Los epígrafes en los que aparecían los nombres tanto del padre como de la hija, (y también de Hortensiano) sufrieron una concienzuda abolitio nominis. Sin embargo, mientras que de la emperatriz conservamos algunos retratos escultóricos, de Plauciano no se conserva ninguno a pesar del testimonio de la Historia Augusta sobre las numerosas esculturas del prefecto ( $\mathrm{Sev}$. 14.5). Según el biógrafo el prefecto perdió momentáneamente el favor del emperador porque había emplazado sus estatuas entre las de los familiares y parientes de Severo por todo el Imperio.

9 LASSÈre 2005, 85.

10 KaJaVa 1994, 136.

11 KaJANTO 1972, 27.

12 Andreu Pintado 2009, 148-149.

13 A partir de la concesión de ciudadanía de Caracalla se constata, sobre todo en aquellas regiones que no habían recibido muchas concesiones con anterioridad, como es el caso de Egipto y Asia, el uso de praenomina sobre todo en un alto porcentaje de notables ciudadanos o funcionarios provinciales. Estos M. Aurelii serían los pertenecientes a las clases altas llegados ahora a la ciudadanía romana a través de la Constitutio. Esa nomenclatura se utilizaba para resaltar un estatus más alto que el que mostraban los simples Aurelii. Esto sería 
excepcional se observó también en Occidente a partir de los Severos. ${ }^{14}$ Un factor adicional que explicaría también la popularidad de los praenomina en el Oriente sería la tradicional importancia del uso de un único nombre en el mundo de habla griega. Además, cabe resaltar el hecho de que muchos nombres polionímicos, tanto de hombres como de mujeres, proceden de África o del Oriente griego. Esta circunstancia podría haber motivado el uso de praenomina femeninos, sobre todo a partir de los siglos II y III. En regiones social y culturalmente ricas, con una cierta atmósfera de "elegancia" podría llegarse a que -en ocasiones- alguna mujer adoptara o añadiera nombres de cara a gozar de un prestigio añadido. ${ }^{15}$ No hay duda de que, en numerosos casos, al menos los praenomina femeninos, fueron un símbolo añadido de estatus que comenzó a ser utilizado ya en una fase avanzada de su vida. No puede ser casualidad que muchas de las mujeres mencionadas a lo largo del trabajo de Mika Kajava tengan una relación muy estrecha con la casa imperial o con el propio emperador. ${ }^{16}$ Parece por tanto que el praenomen era añadido para marcar una nueva posición social cuando se convertía en la mujer del emperador. Claramente muchas de estas damas no habían usado nunca, hasta este momento, sus praenomina. Sin embargo, la circunstancia de que los praenomina de estas mujeres fueron utilizados de forma muy local, y a veces sólo en ocasiones especiales, lo sugiere el hecho de que muchos de ellos son registrados únicamente de forma ocasional o incluso excepcional en inscripciones y monedas.

En los testimonios epigráficos que se han conservado de la emperatriz esposa de Caracalla su nombre aparece como Fulvia Plautilla, o simplemente Plautilla. En ninguno de los que conservamos, a pesar de la damnatio memoriae sufrida, hay rastros de praenomen. De hecho, en una de las primeras publicaciones de marcado carácter científico a principios del siglo XX, concretamente en 1910, Arthur Stein publicó en la Paulys Realencyclopädie der classischen Altertumswissenschaft la biografía de Fulvia Plautilla $:{ }^{17}$ ésta aparece sin el praenomen. Sin embargo, tiempo después, cuando el mismo autor publicó en 1943 la letra F en la Prosopographia Imperii Romani Saec I. II. III., el nombre de la emperatriz ya se muestra como PV(BLIA) FVLVIA PLAVTILLA AVGVSTA ${ }^{18}$ y a partir de este momento cualquier publicación ha mantenido el praenomen en la cita del personaje. ${ }^{19}$

¿Qué pasó entonces entre 1910 y 1943 que motivó la inclusión del praenomen Publia? A tenor de los datos conocidos, en 1910 se publicó en Berlín el volumen Die Antiken Münzen von Dacien und Moesien. Die Münzen von Odessos und Tomis, ${ }^{20}$ que entre sus páginas incluye algunas monedas de Tomis, ciudad de Moesia Inferior, en las que se puede leer un praenomen $\Pi о(\nu \beta \lambda 1 \alpha) / \Pi\left(\right.$ ov $\left.\beta \lambda_{1} \alpha\right)$, referido a la joven emperatriz (Fig. 1). Es verdad que ya entonces eran conocidos otros muchos testi-

una manera visible de marcar las apariencias y daría cierta eminencia a los Marci Aurelii pertenecientes a las clases altas o a los antiguos ciudadanos. Cf. Holtheide 1983, 115-132.

14 LASSÈre 2005, 85.

15 KaJava 1994, 225.

16 Kajava 1994, 225: C. Cornelia Supera (esposa de Emiliano), C. Sulpicia Didymiana (familia de Regaliano), Cnaea Seia... Orbiana (esposa de Alejandro Severo), L. Septimia... Odaenathiana (de la casa real de Palmira), Marcia Annia Latiaria / Appia Annia Elpinice (su padre -Herodes Ático- estaba relacionado con Marco Aurelio); Marcia Otacilia Severa (mujer de Filipo el Árabe), entre otras.

$17 \quad R E$ VII 1, 1910, cols. 285-288, nº 117 (A. Stein).

$18 \quad P I R^{2} \mathrm{~F} 564$

19 V.g. Kienast 2004, 165.

20 IMHOFF-BLuMER 1910, 760-763. 

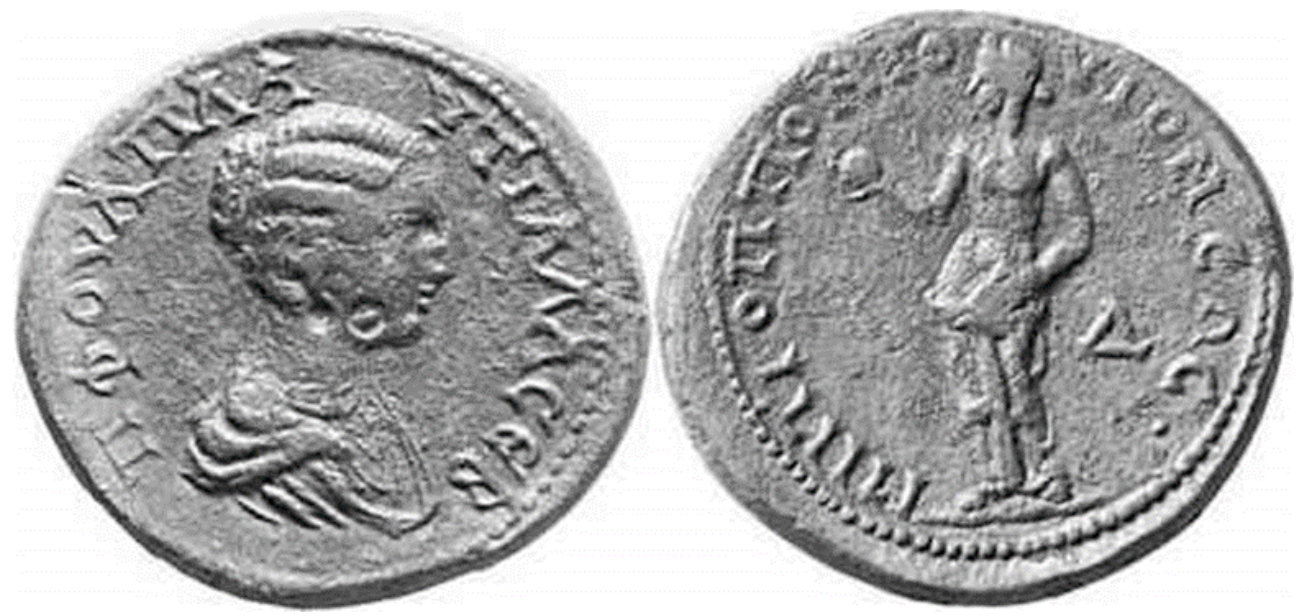

Figura 1. Moneda de Tomis (ImHoff-Blumer 1910, 760, nº 2945).

monios numismáticos en los que la emperatriz aparecía mencionada con su nomen y cognomen o solo cognomen. Sin embargo, hasta el momento no han llegado hasta nosotros otros en los que aparezca el praenomen Publia, por lo que sólo seguimos contando con las monedas de Tomis.

Respecto a este asunto podemos plantearnos algunas preguntas de difícil respuesta. Se podría proponer que realmente la acuñación de Tomis no fue sino un error, o que pudo tratarse de una práctica local que no acabamos de llegar a descubrir o, sencillamente, que fue introducido en esa localidad por razones específicas que a nosotros se nos escapan. ${ }^{21}$ Mika Kajava, refiriéndose a esta cuestión cita como un cierto paralelo ${ }^{22}$ la nomenclatura de Cornelia Salonina, ${ }^{23}$ esposa de Gallieno (253-268)

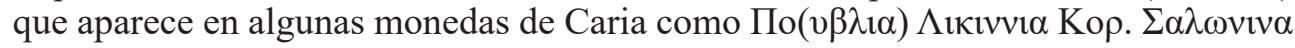
(también en este orden: $\Lambda \mathrm{t}$. По). En este caso parece claro que la nomenclatura la ha tomado de su marido: Publius Licinius Egnatius Gallienus. ${ }^{24}$ Realmente, no creemos que pueda considerarse un paralelo. Asimismo el autor finlandés, en su estudio sobre los praenomina femeninos, alude a las acuñaciones locales de Tesalónica con el praenomen М $\alpha к \iota \alpha$, referido a Antonia la Menor, ${ }^{25}$ hija del triunviro y madre del emperador Claudio, pero apenas es relacionable con el caso que nos ocupa. ${ }^{26}$ No obstante, y esta cuestión es importante para lo que aquí tratamos de plantear, este autor considera que son relativamente frecuentes las anomalías en las estructuras onomásticas en las monedas acuñadas en Oriente y, concretamente, aduce el ejemplo de que Plautilla figura a veces como $\Pi \lambda \alpha v \tau \imath \alpha v \alpha .^{27}$

\footnotetext{
21 KaJAVA 1994, 182.

22 KAJAVA 1994, 182, n 2: “... as a sort of parallel case to Plautilla's praenomen”.

23 Kienast 2004, 222-223.

24 KiENAST 2004, 218-220.

25 Kienast 2004, 88-89.

26 KAJAVA 1994, 182; 168-169 y 224: Es posible que un praenomen fuera asignado a una mujer de rango senatorial porque en ciertos lugares se prefiriera ese tipo, por ejemplo, Marcia Antonia en Tesalónica y P. F. Plautilla en Tomis.

27 KAJAVA 1994, 182.
} 
Respecto a esta cuestión, Percy Gardner en 1875 publicó una nota ${ }^{28}$ en la que ya demostraba que la identificación que se había hecho de la lectura de algunas monedas acerca de una supuesta $\Pi \varepsilon \sigma \chi \varepsilon v v i \alpha ~ \Pi \lambda \alpha v \tau \imath \alpha v \alpha$, identificada además con la esposa de uno de los candidatos al trono tras la muerte del emperador Cómodo, Pescennius Niger, no era tal, sino que se trataba de una acuñación de Fulvia Plautilla, y que los encargados de la acuñación escribieron $\Pi \lambda \alpha v \tau \imath \alpha v \alpha$ posiblemente por asimilación a su padre, Plautianus, ya que, según Gardner, estos ciudadanos de una provincia distante, de Lidia y concretamente de las ciudades de Thyateira y Acrassus,$^{29}$ podrían haber supuesto que tenía el mismo nombre que su padre. ${ }^{30}$ Más adelante incide en lo mismo y, aunque reconoce que podía ser algo inaudito una equivocación en una acuñación de una moneda de la familia imperial, sin embargo, considera que esto se podría explicar porque todas estas monedas fueron acuñadas en Lidia, una "remota" provincia del Imperio. ${ }^{31}$

Es decir, que podríamos plantear que, como en el caso de las ciudades lidias, la acuñación de Tomis podría deberse a una mala interpretación o a un error. Es muy posible que si Plautilla hubiese llevado un praenomen, lo normal es que lo hubiera heredado del padre -como su hermano, Caius Fulvius Hortensianus- por lo que en todo caso nos encontraríamos con una Caia Fulvia Plautilla. Debemos tener en cuenta, además, que los casos que conocemos de otras mujeres de clase alta que llevan praenomen del progenitor: Publia Martia Sergia Fusca, hija o nieta de P. Martius Sergius Saturninus, ${ }^{32}$ cónsul en 198; Publia Valeria Comasia ${ }^{33}$ posible hija de $P$. Valerius Comazon, prefecto del pretorio y cónsul en 220 con Heliogábalo. Existen otros ejemplos también del siglo III. ${ }^{34}$ Ahora bien, también hay que admitir que no siempre es así.

En contraste con los tiempos de la República, en donde el praenomen de la hija en ocasiones es distinto al del padre, ${ }^{35}$ la evidencia epigráfica durante el Imperio muestra que en torno al $90 \%$ de las mujeres cuyo padre es conocido llevan el mismo praenomen que éste. ${ }^{36}$ Concretamente, Kajava comenta como una de las excepciones a esta regla el ejemplo de Plautilla, si bien hace la siguiente salvedad: "but this is a special case, since the praenomen is attested only in Tomis" ${ }^{37}$ En este sentido podríamos citar el caso de otra Augusta que presenta praenomen tanto en los testimonios epigráficos como en los numismáticos que han llegado hasta nosotros. Se trata de la mujer de Alejandro Severo, Gnaea Seia Herennia Sallustia Orba Barbia Orbiana, ${ }^{38}$ hija de L. Seius Herennius Sallustius, ${ }^{39}$ que también, como es evidente, presenta un praenomen distinto del padre..$^{40}$

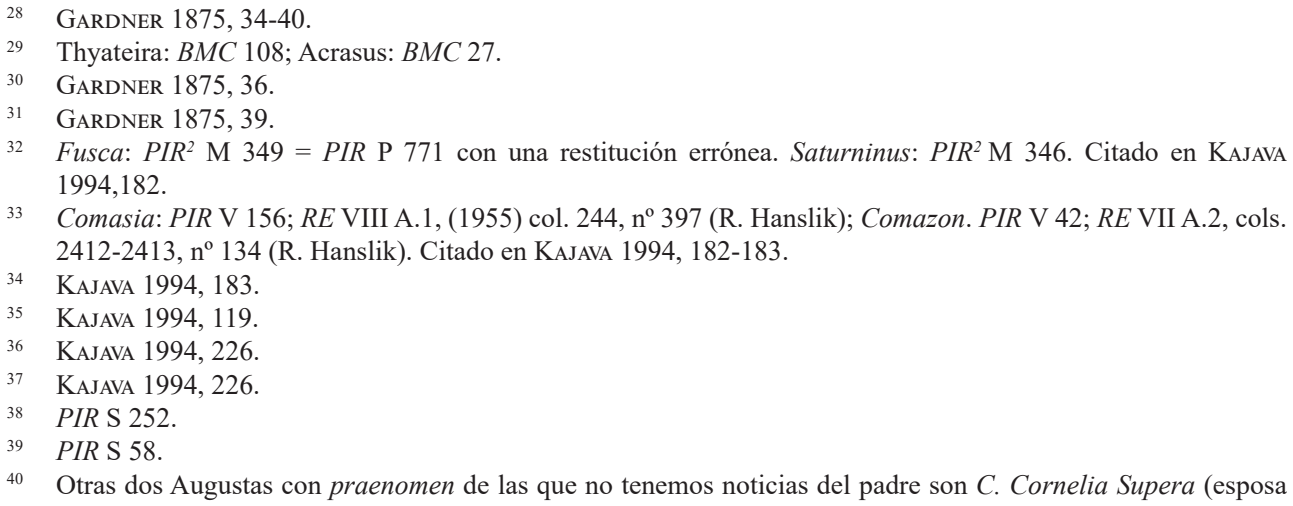


Es muy llamativo que, a pesar del número de referencias epigráficas y sobre todo numismáticas, no haya ninguna otra noticia que haga alusión a este praenomen, salvo las procedentes de Tomis. Pensamos que, por la misma razón que no tenemos en consideración el cognomen $\Pi \lambda \alpha v \tau \imath \alpha v \alpha$ que aparece en las monedas de las ciudades lidias citadas anteriormente, tampoco hay razones suficientes para suponer que su praenomen fuese Publia.

En último lugar, también queremos llamar la atención sobre otro praenomen que ha sido atribuido a Plautilla: el de Iusta (Iusta Fulvia Plautilla). El testimonio más antiguo de dicha identificación es de Guillaume Beauvais quien, en una de sus obras de $1767,{ }^{41}$ llama a la emperatriz de esta forma. ${ }^{42}$ En este caso no se conoce absolutamente ninguna otra evidencia, ésta es la mención más antigua. Desconocemos si el primero fue Beauvais o si éste, a su vez, lo tomó de otra fuente desconocida. Otros autores posteriores han repetido este nombre manteniendo el error hasta la actualidad..$^{43}$ En cualquier caso no hay ninguna razón para seguir utilizando este praenomen, puesto que no existe ningún documento literario, ni epigráfico, ni numismático coetáneo a la emperatriz que justifique tal uso.

\section{Referencias bibliográficas}

Andreu Pintado, J. (2009): “La onomástica individual”, [en] J. Andreu Pintado (coord.), Fundamentos de Epigrafía Latina, Madrid, 143-173.

BeAuvais, G. (1767): Histoire abrégée des empereurs romains et grecs, des impératrices et des Césars, des tyrans et des personnes des familles impériales pour lesquelles on a frappé des médailles, depuis Pompée jusqu'à la prise de Constantinople par les Turcs, sous Constantin XIV, dernier Empereur Grec, Paris [Académie des sciences, belles-lettres et arts de Bure père].

Birley, A. (2012): Septimio Severo. El emperador africano, Madrid.

CAliXte de PÉNA, J. F. (1823): Leçons élémentaires de Numismatique Romaine, puisées dans l'examen d'une collection particulière, Paris.

CAYón, J. R. (1985): Compendio de las Monedas del Imperio Romano, Vol. II. De Caracalla, 198 d.C., a Juliano de Pannonia, 285 d.C., Madrid.

Christol, M. (1997): “L'épigraphie de Thugga et la carrière de Plautien”, [en] M. Khanoussi - L. Maurin (eds.), Dougga (Thugga). Etudes épigraphiques, Paris, 127-140.

Christol, M. - Drew-Bear, Th. (1995): “Q. Aurelius Polus Terentianus et Q. Hedius Rufus Lollianus Gentianus, proconsuls d'Asie”, Anatolia Antiqua 3/1, 67-93.

de Emiliano), en este caso sólo por testimonios numismáticos; de Marcia Otacilia Severa (mujer de Filipo el Árabe) tenemos una noticia de Zósimo, que en su Historia Romana (3.19.2) menciona a un tal Severianus (PIR S 440) como padre o hermano.

41 BeAuvais 1767, 307-309. Este autor fue un conocido historiador francés del siglo XVIII, cuya especialidad eran fundamentalmente las monedas.

42 Sólo conocemos una mujer de familia imperial que portaba este praenomen: Iusta Grata Honoria, hija de Constancio III y Galla Placidia. Sobre este praenomen cf. KaJAVA 1994, 33 y 42.

43 Desde el siglo XVIII, entre otros: DAnTIne et alii 1770, 342; PeIGNOt 1804, 44; CraBB 1825, sin numerar, voz Plautilla; PonzóA - Bober de Roselló 1846, 247; Glaire et alii 1848. En la actualidad siguen utilizando la forma Iusta Fulvia “Plautilla” CAYÓN 1985, 825, y CRESPO PÉREZ 2014, 43. 
Conesa Navarro, P. D. - González Fernández, R. (2016): “Fuluia Plautilla. Instrumento legitimador y político de la dinastía y del prefecto del pretorio”, Athenaeum 104/1, 129 156.

CRABB, G. (1825): Universal historical dictionary; or, Explanation of the names of persons and places in the departments of Biblical, political, and ecclesiastical history, mythology, heraldry, biography, bibliography, geography, and numismatics. Illustrated by very numerous portraits and medallic cuts, London, vol. 2.

Crespo PÉRez, C. (2014): La condenación al olvido (damnatio memoriae). La deshonra pública tras la muerte en la política romana (siglos I - IV d.C.), Madrid-Salamanca.

Daguet-Gagey, A. (2000): Septime Severe. Rome, l'Afrique et l'Orient, Paris.

Dantine, M.-F. - Durand, U. - Clémencet, Ch. (DOM) - Clément, F. (DOM), (1770): L'art de vérifier les dates des faits historiques, des chartes, des chroniques et autres anciens monuments, depuis la naissance de Notre-Seigneur par le moyen d'un table chronologique, Paris.

GARDNer, P. (1875): "Plautiana. A Rectification", The Numismatic Chronicle and Journal of the Numismatic Society, New Series 15, 34-40.

Glaire, J. B. - Chantrel, J. - Walsh, J. A. - Alletz, E. (1848): Encyclopédie catholique, répertoire universel et raisonné des sciences, des lettres, des arts et des métiers, formant une bibliothèque universelle, avec la biographie des hommes célèbres: ornée de plus de 3000 gravures dans le texte et refermant le résumé de plus de dix mille ouvrages, Paris, vol. 16.

González Fernández, R. - Conesa Navarro, P. D.

(2014): "Plauciano: la amenaza de la domus severiana", Potestas 7, 27-50.

(e.p.): "Fulvia Plautilla sponsa Marci Aureli Antonini et Augusta. Sobre la política dinástica de Septimio Severo".

HoltheIDE, B. (1983): Römische Bürgerrechtspolitik und römische Neubürger in der Provinz Asia, Freiburg.

Imhoof-Blumer, F. (1910): Die antiken Münzen Nord-Griechenlands. Band 1: Die antiken Münzen von Dacien und Moesien, bearbeitet von Behrendt Pick und Kurt Regling, Halbband 2, Abt. 1 Die Münzen von Odessos und Tomis, Berlin.

KajANTO, I. (1972): “Women's praenomina reconsidered”, Arctos 7, 13-30.

KajaVA, M. (1994): Roman Female Praenomina. Studies in the Nomenclature of Roman Women, Rome.

KiEnast, D. (2004): Römische Kaisertabelle. Grundzüge einer römischen Kaiserchronologie, Darmstadt.

Lassère, J. M. (2005): Manuel d'Épigraphie Romaine, Paris, vol. I.

Peignot, G. (1804): Essai de curiosités bibliographiques, Paris.

Ponzóa, F. - Bober de Roselló, J. M. (1846): Diccionario manual para el estudio de antigüedades, Palma de Mallorca [Imprenta de Pedro José Gelabert]. 